\title{
Nearly equal distances in the plane
}

Paul Erdős*, Endre Makai* and János Pach**

\begin{abstract}
For any positive integer $k$ and $\epsilon>0$, there exist $n_{k, \epsilon}, c_{k, \epsilon}>0$ with the following property. Given any system of $n>n_{k, \epsilon}$ points in the plane with minimal distance at least 1 and any $t_{1}, t_{2}, \ldots, t_{k} \geq 1$, the number of those pairs of points whose distance is between $t_{i}$ and $t_{i}+c_{k, \epsilon} \sqrt{n}$ for some $1 \leq i \leq k$, is at most $\frac{n^{2}}{2}\left(1-\frac{1}{k+1}+\epsilon\right)$. This bound is asymptotically tight.
\end{abstract}

\section{Introduction}

Almost fifty years ago the senior author [E1] raised the following problem: Given $n$ points in the plane, what can be said about the distribution of the $\left(\begin{array}{l}n \\ 2\end{array}\right)$ distances determined by them? In particular, what is the maximum number of pairs of points that determine the same distance? Although a lot of progress has been made in this area, we are still very far from having satisfactory answers to the above questions (cf. $[\mathrm{EP}],[\mathrm{MP}],[\mathrm{PA}]$ for recent surveys).

Two distances are said to be nearly the same if they differ by at most 1 . If all points of a set are close to each other, then all distances determined by them are nearly the same (nearly zero). Therefore, throughout this paper we shall consider only separated point sets $P$, i.e., we shall assume that the minimal distance between two elements of $P$ is at least 1. In [EMPS] we have shown that the maximum number of times that nearly the same distance can occur among $n$ separated points in the plane is $\left\lfloor n^{2} / 4\right\rfloor$, provided that $n$ is sufficiently large. In fact, a straightforward generalization of our argument gives the following.

Theorem 1. There exists $c_{1}>0$ and $n_{1}$ such that, for any set $\left\{p_{1}, p_{2}, \ldots, p_{n}\right\} \subseteq \mathbb{R}^{2}$ $\left(n \geq n_{1}\right)$ with minimal distance at least 1 and for any real $t$, the number of pairs $\left\{p_{i}, p_{j}\right\}$

\footnotetext{
* Mathematical Institute of the Hungarian Academy of Sciences

** Dept. Computer Sci., City College, New York and Mathematical Institute of the Hungarian Academy of Sciences. Research supported by NSF grant CCR-91-22103 and OTKA-1907 and 4269.
} 
whose distance $d\left(p_{i}, p_{j}\right) \in\left[t, t+c_{1} \sqrt{n}\right]$ is at most $\left[n^{2} / 4\right]$. (Evidently, the statement is false with, say, $\left.c_{1}=2.\right)$

The aim of the present note is to establish the following result.

Theorem 2. Given any positive integer $k$ and $\epsilon>0$, one can find a function $c(n)$ tending to infinity and an integer $n_{0}$ satisfying the following condition. For any set $\left\{p_{1}, p_{2}, \ldots, p_{n}\right\} \subseteq \mathbb{R}^{2}\left(n \geq n_{0}\right)$ and for any reals $t_{1}, t_{2}, \ldots, t_{k}$, the number of pairs $\left\{p_{i}, p_{j}\right\}$ whose distance

$$
d\left(p_{i}, p_{j}\right) \in \bigcup_{r=1}^{k}\left[t_{r}, t_{r}+c(n)\right]
$$

is at most $\frac{n^{2}}{2}\left(1-\frac{1}{k+1}+\epsilon\right)$.

To see that this bound is asymptotically tight, let $P=\{(i N, j): 0 \leq i \leq k, 1 \leq j \leq$ $\left.\frac{n}{k+1}\right\}$, where $N$ is a very large constant. Now $|P| \leq n$, and the distance between any two points of $P$ with different $x$-coordinates is nearly $i N$ for some $1 \leq i \leq k$. Hence, there are at least $\frac{n^{2}}{2}\left(1-\frac{1}{k+1}+o(1)\right)$ point pairs such that all distances determined by them belong to the union of the intervals $[i N, i N+1], 1 \leq i \leq k$.

Let $K_{k+2}^{(m)}$ denote a $(k+2)$-uniform hypergraph, whose vertex set can be partitioned into $k+2$ parts $V\left(K_{k+2}^{(m)}\right)=V_{1} \cup V_{2} \cup \cdots \cup V_{k+2},\left|V_{i}\right|=m(1 \leq i \leq k+2)$, and $K_{k+2}^{(m)}$ consists of all $(k+2)$-tuples containing exactly one point from each $V_{i}$. Our proof is based on the following two well-known facts from extremal (hyper)graph theory.

Theorem A $\left[\mathbf{L}\right.$, Ch. 10, Ex. 40]. Any graph with $n$ vertices and $\frac{n^{2}}{2}\left(1-\frac{1}{k+1}+\epsilon\right)$ edges has at least $\epsilon\left(\frac{n}{k+2}\right)^{k+2}$ complete subgraphs on $k+2$ vertices.

Theorem B [E2]. Any $(k+2)$-uniform hypergraph with $n$ vertices and at least $n^{k+2-(1 / m)^{k+1}}$ hyperedges contains a subhypergraph isomorphic to $K_{k+2}^{(m)}$.

In the last section we are going to show that Theorem 2 is valid with $c(n)=c_{k, \epsilon} \sqrt{n}$, for a suitable constant $c_{k, \epsilon}>0$. Our main tool will be a straightforward generalization of Szemerédi's Regularity Lemma. Given a graph $G$ whose edges are colored by $k$ colors, 
and two disjoint subsets $V_{1}, V_{2} \subseteq V(G)$, let $e_{r}\left(V_{1}, V_{2}\right)$ denote the number of edges of color $r$ with one endpoint in $V_{1}$ and the other in $V_{2}$. The pair $\left\{V_{1}, V_{2}\right\}$ is called $\delta$-regular if

$$
\left|\frac{e_{r}\left(V_{1}^{\prime}, V_{2}^{\prime}\right)}{\left|V_{1}^{\prime}\right| \cdot\left|V_{2}^{\prime}\right|}-\frac{e_{r}\left(V_{1}, V_{2}\right)}{\left|V_{1}\right| \cdot\left|V_{2}\right|}\right|<\delta \quad \text { for every } 1 \leq r \leq k,
$$

and for every $V_{1}^{\prime} \subseteq V_{1}, V_{2}^{\prime} \subseteq V_{2}$ such that $\left|V_{1}^{\prime}\right| \geq \delta\left|V_{1}\right|,\left|V_{2}^{\prime}\right| \geq \delta\left|V_{2}\right|$. We say that the sizes of $V_{1}$ and $V_{2}$ are almost equal if ||$V_{1}|-| V_{2}|| \leq 1$.

Theorem $\mathbf{C}[\mathbf{S z}]$. Given any $\delta>0$ and any positive integers $k, f$, there exist $F=$ $F(\delta, k, f)$ and $n_{0}=n_{0}(\delta, k, f)$ with the property that the vertex set of every graph $G$ with $|V(G)|>n_{0}$, whose edges are colored by $k$ colors, can be partitioned into almost equal classes $V_{1}, V_{2}, \ldots, V_{g}$ such that $f \leq g \leq F$ and all but at most $\delta g^{2}$ pairs $\left\{V_{i}, V_{j}\right\}$ are $\delta$-regular.

\section{Proof of Theorem 2}

The proof is by induction on $k$. For $k=1$ the assertion is true (Theorem 1). So we can assume that $k \geq 2, \epsilon>0$, and that we have already proved the theorem for $k-1$ with an appropriate function $c_{k-1, \epsilon}(n) \rightarrow \infty$.

Fix a set $P=\left\{p_{1}, p_{2}, \ldots, p_{n}\right\} \subseteq \mathbb{R}^{2}$ with minimal distance 1 , and suppose that there are reals $t_{1}, t_{2}, \cdots, t_{k}$ such that the number of pairs $\left\{p_{i}, p_{j}\right\}$ with

$$
d\left(p_{i}, p_{j}\right) \in \bigcup_{r=1}^{k}\left[t_{r}, t_{r}+c(n)\right]
$$

is at least $\frac{n^{2}}{2}\left(1-\frac{1}{k+1}+\epsilon\right)$. We are going to show that one can specify the function $c(n) \leq c_{k-1, \epsilon}(n)$ tending to infinity so as to obtain a contradiction if $n$ is sufficiently large.

Lemma 2.1. If $c(n)=o(\sqrt{n})$, then $\min _{1 \leq r \leq k} t_{r} / \sqrt{n} \rightarrow \infty$ as $n$ tends to infinity.

Proof. Assume that e.g. $t_{k} \leq C \sqrt{n}$. For any $p_{i}$, the number of points $p_{j}$ with $d\left(p_{i}, p_{j}\right) \in$ $\left[t_{k}, t_{k}+c(n)\right]$ is at most $100\left(t_{k}+c(n)\right) c(n)$. Hence the number of point pairs whose distances belong to $\bigcup_{r=1}^{k-1}\left[t_{r}, t_{r}+c_{k-1, \epsilon}(n)\right] \supseteq \bigcup_{r=1}^{k-1}\left[t_{r}, t_{r}+c(n)\right]$ is at least

$$
\frac{n^{2}}{2}\left(1-\frac{1}{k+1}+\epsilon\right)-50 n\left(t_{k}+c(n)\right) c(n)>\frac{n^{2}}{2}\left(1-\frac{1}{k}+\epsilon\right),
$$


provided that $n$ is sufficiently large. This contradicts the induction hypothesis.

Lemma 2.2. One can choose disjoint subsets $P_{i} \subseteq P(1 \leq i \leq k+2)$ such that $\left|P_{i}\right|>$ $b_{k, \epsilon}(\log n)^{1 /(k+1)}$ for a suitable constant $b_{k, \epsilon}>0$, and the following condition holds. For any $1 \leq i \neq j \leq k+2$, there exists $1 \leq r(i, j) \leq k$ such that

$$
d\left(p_{i}, p_{j}\right) \in\left[t_{r(i, j)}, t_{r(i, j)}+c(n)\right] \quad \text { for all } p_{i} \in P_{i}, p_{j} \in P_{j}
$$

Proof. Let $G$ denote the graph with vertex set $P$, whose two vertices are connected by an edge if and only if their distance belongs to $\bigcup_{r=1}^{k}\left[t_{r}, t_{r}+c(n)\right]$. By Theorem A (in the Introduction), we obtain that $G$ contains at least $\epsilon\left(\frac{n}{k+2}\right)^{k+2}$ complete subgraphs $K_{k+2}$ on $k+2$ vertices. Since for a random partition $\left\{P_{1}, \ldots, P_{k+2}\right\}$ of $P$ the number of the above $K_{k+2}$ 's meeting each $P_{i}$ in one point is at least $d(k) \epsilon(n /(k+2))^{k+2}$, where $d(k)>0$ we can suppose this inequality for a fixed partition $\left\{P_{1}, \ldots, P_{k+2}\right\}$ of $P$.

Let $K_{k+2}$ be such a subgraph with vertices $p_{s_{1}}, p_{s_{2}}, \ldots, p_{s_{k+2}}\left(1 \leq s_{1}<s_{2}<\cdots<\right.$ $\left.s_{k+2} \leq n\right)$. Then for any $1 \leq i \neq j \leq k+2$, there exists $1 \leq r(i, j) \leq k$ such that $d\left(p_{s_{i}}, p_{s_{j}}\right) \in\left[t_{r(i, j)}, t_{r(i, j)}+c(n)\right]$. The symmetric array $(r(i, j))_{1 \leq i \neq j \leq k+2}$ is said to be the

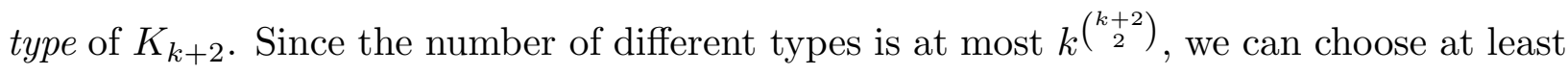
$\frac{\epsilon}{k^{(k+2)^{2}}} n^{k+2}$ complete subgraphs $K_{k+2}$ having the same type. Applying Theorem B to the $(k+2)$-uniform hypergraph $H$ formed by the vertex sets of these complete subgraphs, we obtain that $H$ contains a subhypergraph isomorphic to $K_{k+2}^{(m)}$ with $m \geq b_{k, \epsilon}^{\prime}(\log n)^{1 /(t+2)}$, for a suitable constant $b_{k, \epsilon}^{\prime}>0$. From this the assertion readily follows.

In what follows, we shall analyze the relative positions of the sets $P_{i}(1 \leq i \leq k+2)$ described in Lemma 2.2. Consider two sets, $P_{1}$ and $P_{2}$ (say), and assume that all distances between them belong to the interval $\left[t_{1}, t_{1}+c(n)\right]$. For any $p, p^{\prime} \in P_{1}$, all elements of $P_{2}$ must lie in the intersection of two annuli centered at $p$ and $p^{\prime}$. If $d\left(p, p^{\prime}\right)<2 t_{1}$, $c(n)=o(\sqrt{n})$, then (by Lemma 2.1) the area of this intersection set is at most

$$
\frac{20 t_{1}^{2} c^{2}(n)}{d\left(p, p^{\prime}\right) \sqrt{4 t_{1}^{2}-d^{2}\left(p, p^{\prime}\right)}},
$$


and, using the notation $m(n)=b_{k, \epsilon}(\log n)^{1 /(t+1)}$, we have

$$
m(n) \leq\left|P_{2}\right| \leq \frac{50 t_{1}^{2} c^{2}(n)}{d\left(p, p^{\prime}\right) \sqrt{4 t_{1}^{2}-d^{2}\left(p, p^{\prime}\right)}} .
$$

Assuming that $c(n)=o(\sqrt{m(n)})$, this immediately implies that $d\left(p, p^{\prime}\right) / t_{1}$ is either close to 0 or close to 2 . More exactly,

$$
d\left(p, p^{\prime}\right) \in\left[1, \frac{50 c^{2}(n)}{m(n)} t_{1}\right] \cup\left[\left(2-\frac{50 c^{2}(n)}{m(n)}\right) t_{1}, 2 t_{1}+2 c(n)\right]
$$

for any $p, p^{\prime} \in P_{1}$, provided that $n$ is large enough.

Pick now any point $q \in P_{2} . P_{1}$ must be entirely contained in the annulus around $q$, whose inner and outer radii are $t_{1}$ and $t_{1}+c(n)$, respectively. Thus, if $P_{1}$ has two elements with $d\left(p, p^{\prime}\right) \geq\left(2-\frac{50 c^{2}(n)}{m(n)}\right) t_{1}$, then all other points of $P_{1}$ must lie in the union of the two circles of radius $\frac{50 c^{2}(n)}{m(n)} t_{1}$ centered at $p$ and $p^{\prime}$. In any case, there is an at least $\frac{m(n)}{2}$-element subset $P_{1}^{\prime} \subseteq P$, whose diameter

$$
\operatorname{diam} P_{1}^{\prime} \leq \frac{50 c^{2}(n)}{m(n)} t_{1}=o(1) t_{1}
$$

Repeating this argument ( $k+2$ times), we obtain the following

Lemma 2.3. Let $m(n)=b_{k, \epsilon}(\log n)^{1 /(k+1)}, c(n)=o(\sqrt{m(n)})$. Then one can choose disjoint subsets $Q_{i} \subseteq P,\left|Q_{i}\right| \geq m(n) / 2(1 \leq i \leq k+2)$ such that the following conditions are satisfied.

(i) For any $1 \leq i \neq j \leq k+2$, there exists $1 \leq r(i, j) \leq k$ such that

$$
d\left(p_{i}, p_{j}\right) \in\left[t_{r(i, j)}, t_{r(i, j)}+c(n)\right] \quad \text { for all } \quad p_{i} \in Q_{i}, \quad p_{j} \in Q_{j}
$$

(ii) For any $1 \leq i \leq k+2$,

$$
\operatorname{diam} Q_{i}=o(1) \min _{j \neq i} t_{r(i, j)}
$$

(iii) There is a line $\ell$ such that the angle between $\ell$ and any line $p_{i} p_{j}\left(p_{i} \in Q_{i}, p_{j} \in Q_{j}\right.$, $i \neq j)$ is $o(1)$. 
Proof. We only have to prove part (iii). Fix two subsets $Q_{i}$ and $Q_{j}(i \neq j)$. By (ii),

$$
\max \left(\operatorname{diam} Q_{i}, \operatorname{diam} Q_{j}\right)=o(1) t_{r(i, j)}
$$

so the angle between any two lines $p_{i} p_{j}$ and $p_{i}^{\prime} p_{j}^{\prime}\left(p_{i}, p_{i}^{\prime} \in Q_{i} ; p_{j}, p_{j}^{\prime} \in Q_{j} ; i \neq j\right)$ is $o(1)$.

Let $q_{i}$ and $q_{i}^{\prime}$ be wo elements of $Q_{i}$ whose distance is maximal. Clearly, for any $j \neq i$,

$$
\frac{\sqrt{m(n)}}{10} \leq d\left(q_{i}, q_{i}^{\prime}\right)=\operatorname{diam} Q_{i} \leq o(1) t_{r(i, j)}
$$

It is sufficient to show that, for any $p_{j} \in Q_{j}$, the lines $q_{i} q_{i}^{\prime}$ and $q_{i} p_{j}$ are almost perpendicular. Indeed.

$$
\begin{aligned}
\left|\cos \left(\angle q_{i}^{\prime} q_{i} p_{j}\right)\right| & =\left|\frac{\left(d\left(q_{i}, p_{j}\right)-d\left(q_{i}^{\prime}, p_{j}\right)\right)\left(d\left(q_{i}, p_{j}\right)+d\left(q_{i}^{\prime}, p_{j}\right)\right)+d^{2}\left(q_{i}, q_{i}^{\prime}\right)}{2 d\left(q_{i}, p_{j}\right) d\left(q_{i}, q_{i}^{\prime}\right)}\right| \\
& \leq \frac{c(n)\left(2 t_{r(i, j)}+2 c(n)\right)}{2 t_{r(i, j)}(\sqrt{m(n)} / 10)}+\frac{d\left(q_{i}, q_{i}^{\prime}\right)}{2 t_{r(i, j)}}=o(1) .
\end{aligned}
$$

We need the following key property of the sets $Q_{i}$ constructed above.

Lemma 2.4. Let $s \geq 3$ be fixed, and suppose that

$$
\operatorname{diam}\left(Q_{1} \cup Q_{2} \cup \cdots \cup Q_{s}\right)=d\left(p_{1}, p_{2}\right) \quad \text { for some } p_{1} \in Q_{1}, p_{2} \in Q_{2}
$$

Then, for any $1 \leq i \neq j \leq s, r(i, j)=r(1,2)$ if and only if $\{i, j\}=\{1,2\}$.

Proof. Suppose, in order to obtain a contradiction, that there are two points $p_{i}^{\prime} \in Q_{i}$, $p_{j}^{\prime} \in Q_{j}, 2 \leq i \neq j \leq s$ such that

$$
d\left(p_{i}^{\prime}, p_{j}^{\prime}\right) \in\left[t_{r(1,2)}, t_{r(1,2)}+c(n)\right]
$$

By Lemma 2.1 and Lemma 2.3 (iii), all points of $Q_{2} \cup Q_{3} \cup \ldots \cup Q_{5}$ lie in a small sector (of angle $o(1)$ ) of the annulus around $p_{1}$, whose inner and outer radii are $\sqrt{n}$ and $d\left(p_{1}, p_{2}\right)$, respectively. Obviously, the diameter of this sector is $d(u, v)$, where $u(\operatorname{resp} . v)$ is the 
intersection of one (the other) boundary ray with the inner (outer) circle of the annulus. But then we have

$$
\begin{aligned}
d\left(p_{1}, p_{2}\right)-d\left(p_{i}^{\prime}, p_{j}^{\prime}\right) & \geq d\left(p_{1}, p_{2}\right)-d(u, v)=d\left(p_{1}, v\right)-d(u, v) \\
& =\frac{2 d\left(p_{1}, u\right) d\left(p_{1}, v\right) \cos \left(\angle u p_{1} v\right)-d^{2}\left(p_{1}, u\right)}{d\left(p_{1}, v\right)+d(u, v)} \\
& \geq d\left(p_{1}, u\right) \cos \left(\angle u p_{1} v\right)-\frac{d^{2}\left(p_{1}, u\right)}{2 d\left(p_{1}, v\right)} \\
& \geq \sqrt{n}(1-o(1))-\frac{n}{2 t_{r(1,2)}}>\frac{\sqrt{n}}{2}>c(n)
\end{aligned}
$$

the desired contradiction.

Now we can easily complete the proof of Theorem 2. For the sake of simplicity we assume the intervals are disjoint, but the same arguments work in the general case as well. Assume without loss of generality that the diameter of $Q=Q_{1} \cup Q_{2} \cup \cdots \cup Q_{k+2}$ is attained between a point of $Q_{1}$ and a point of $Q_{j_{1}}$, for some $j_{1}>1$. By Lemma 2.4, no distance determined by the set $Q^{\prime}=Q_{2} \cup Q_{3} \cup \cdots \cup Q_{k+2}$ belongs to the interval $\left[t_{r\left(1, j_{1}\right)}, t_{r\left(1, j_{1}\right)}+c(n)\right]$. Suppose that the diameter of $Q^{\prime}$ is attained between a point of $Q_{2}$ and a point of $Q_{j_{2}}, j_{2}>2$. Applying the lemma again, we obtain that none of the distances determined by $Q^{\prime \prime}=Q_{3} \cup Q_{4} \cup \cdots \cup Q_{k+2}$ is in $\left[t_{r\left(2, j_{2}\right)}, t_{r\left(2, j_{2}\right)}+c(n)\right]$, where $r\left(2_{1 j_{2}}\right) \neq r\left(1_{1 j_{1}}\right)$. Proceeding like this, we can conclude that no distance determined by $Q_{k+1} \cup Q_{k+2}$ belongs to

$$
\bigcup_{i=1}^{k}\left[t_{r\left(i, j_{i}\right)}, t_{r\left(i, j_{i}\right)}+c(n)\right],
$$

where $\left\{r\left(i, j_{i}\right): 1 \leq i \leq k\right\}=\{1,2, \cdots, k\}$. In other words, there exists no integer $r(k+$ $1, k+2)$ satisfying the condition in Lemma 2.3 (i). This contradiction completes the proof of Theorem 2 for any function $c(n)=o\left((\log n)^{1 /(2 k+2)}\right)$. In fact, our argument also shows that there is a small constant $c_{k, \epsilon}>0$ such that the theorem is true with $c(n)=c_{k, \epsilon}(\log n)^{1 /(2 k+2)}$. 


\section{Strengthening of Theorem 2}

In this section we are going to modify the above arguments to show that Theorem 2 is valid for any function $c(n)=o(\sqrt{n})$. Notice that in the previous section we have not really used the fact that all distances between $Q_{i}$ and $Q_{j}$ (in Lemma 2.3) belong to the interval $\left[t_{r(i, j)}, t_{r(i, j)}+c(n)\right]$. It is sufficient to require that many distances have this property, and there are much larger subsets $Q_{i}(1 \leq i \leq k+2)$ satisfying this weaker condition. As a matter of fact, we can assume that $\left|Q_{i}\right| \geq m(n)=b_{k, \epsilon}^{*} n$ for a suitable constant $b_{k, \epsilon}^{*}>0$, and follow essentially the same argument as before for any $c(n)=o(\sqrt{m(n)})=o(\sqrt{n})$.

In the following we shall assume that $k, \epsilon$ and $\delta<\left(\frac{\epsilon}{100 k}\right)^{k+5}$ are fixed, $c(n)=o(\sqrt{n})$, and $n$ is very large. We want to apply Theorem $\mathrm{C}$ (in the Introduction) to the graph $G$ on the vertex set $P$, whose two points $p, p^{\prime}$ are connected by an edge of color $r$ whenever

$$
d\left(p, p^{\prime}\right) \in\left[t_{r}, t_{r}+c(n)\right], \quad 1 \leq r \leq k
$$

and $r$ is minimal with this property. Then Lemma 2.3 can be replaced by the following.

Lemma 3.1. There is a constant $b=b(k, \epsilon, \delta)$ such that one can find disjoint subsets $Q_{i} \subseteq P,\left|Q_{i}\right|>$ bn $(1 \leq i \leq k+2)$ satisfying the following conditions.

(i) For any $1 \leq i \neq j \leq k+2$, there exists $1 \leq r(i, j) \leq k$ such that

$$
\frac{e_{r(i, j)}\left(Q_{i}, Q_{j}\right)}{\left|Q_{i}\right| \cdot\left|Q_{j}\right|} \geq \frac{\epsilon}{20 k}
$$

(ii) For any $1 \leq i \leq k+2$,

$$
\operatorname{diam} Q_{i}=o(1) \min _{j \neq i} t_{r(i, j)} ;
$$

(iii) There is a line $\ell$ such that the angle between $\ell$ and any line $p_{i} p_{j}\left(p_{i} \in Q_{i}, p_{j} \in Q_{j}\right.$, $\left.p_{i} p_{j} \in E(G)\right)$ is o(1).

Proof. Consider a partition $V(G)=P=V_{1} \cup V_{2} \cup \cdots \cup V_{g}$ meeting the requiremens of Theorem $\mathrm{C}$ with $f=\lceil 10 / \epsilon\rceil$. Let $G^{*}$ denote the graph with vertex set $V\left(G^{*}\right)=$ $\left\{V_{1}, V_{2}, \ldots, V_{g}\right\}$, where $V_{i}$ and $V_{j}$ are joined by an edge if $\left\{V_{i}, V_{j}\right\}$ is a $\delta$-regular pair and

$$
\frac{e_{r(i, j)}\left(V_{i}, V_{j}\right)}{\left|V_{i}\right| \cdot\left|V_{j}\right|} \geq \frac{\epsilon}{10 k}
$$


for some $1 \leq r(i, j) \leq k$. Clearly,

$$
\frac{n^{2}}{2}\left(1-\frac{1}{k+1}+\epsilon\right) \leq|E(G)| \leq\left(\left|E\left(G^{*}\right)\right|+\delta g^{2}+\left(\begin{array}{c}
g \\
2
\end{array}\right) \frac{\epsilon}{10}\right)\left\lceil\frac{n}{g}\right\rceil^{2}+g\left(\begin{array}{c}
\lceil n / g\rceil \\
2
\end{array}\right)
$$

whence

$$
\left|E\left(G^{*}\right)\right| \geq \frac{g^{2}}{2}\left(1-\frac{1}{k+1}+\epsilon / 2\right)
$$

By Theorem A (or by Turán's theorem [T]), this implies that $G^{*}$ has a complete subgraph on $k+2$ vertices, say, $V_{1}, V_{2}, \ldots, V_{k+2}$.

Assume without loss of generality that $r(1,2)=1, t_{1}=\min _{j \neq 1} t_{r(1, j)}$, and let $G_{r}$ denote the subgraph of $G$ consisting of all edges of color $r$. By (1), at least $\frac{\epsilon}{10 k}\left|V_{1}\right| \cdot\left|V_{2}\right|$ edges of $G_{1}$ run between $V_{1}$ and $V_{2}$. Therefore, we can pick a point $p_{2} \in V_{2}$ connected to all elements of a subset $P_{1} \subseteq V_{1},\left|P_{1}\right| \geq \frac{\epsilon}{10 k}\left|V_{1}\right|$. Clearly, $P_{1}$ lies in an annulus centered at $p_{2}$ with inner radius $t_{1}$ and outer radius $t_{1}+c(n)$. Using the fact that $\left\{V_{1}, V_{2}\right\}$ is a $\delta$-regular pair, it can be shown by routine calculations that there are $\left(\frac{\epsilon}{100 k}\right)^{4}\left|P_{1}\right|^{2}$ pairs $\left\{p_{1}, p_{1}^{\prime}\right\} \subset P_{1}$ such that $p_{1}$ and $p_{1}^{\prime}$ have at least $\left(\frac{\epsilon}{100 k}\right)^{2}\left|V_{2}\right| \geq \frac{1}{F(\delta, k, f)}\left(\frac{\epsilon}{100 k}\right)^{2} n$ common neighbors in $G_{1}$. As in the proof of Lemma 2.3, we can argue that, for any such pair,

$$
d\left(p_{1}, p_{1}^{\prime}\right)=o(1) t_{1} \quad \text { or } \quad d\left(p_{1}, p_{1}^{\prime}\right)=(2-o(1)) t_{1} .
$$

Hence, we can find a point $p_{1} \in P_{1}$ such that

$$
\left|\left\{p_{1}^{\prime} \in P_{1}: d\left(p_{1}, p_{1}^{\prime}\right)=o(1) t_{1}\right\}\right| \geq\left(\frac{\epsilon}{100 k}\right)^{4}\left|P_{1}\right|,
$$

or

$$
\left|\left\{p_{1}^{\prime} \in P_{1}: d\left(p_{1}, p_{1}^{\prime}\right)=(2-o(1)) t_{1}\right\}\right| \geq\left(\frac{\epsilon}{100 k}\right)^{4}\left|P_{1}\right|
$$

Let $Q_{1} \subseteq P_{1}$ denote the larger of these two sets. Then

$$
\left|Q_{1}\right| \geq\left(\frac{\epsilon}{100 k}\right)^{4}\left|P_{1}\right|>\left(\frac{\epsilon}{100 k}\right)^{5}\left|V_{1}\right| \geq \frac{1}{F(\delta, k, f)}\left(\frac{\epsilon}{100 k}\right)^{5} n
$$

and diam $Q_{1}=o(1) t_{1}$. Repeating the same argument for every $V_{i}(1 \leq i \leq k+2)$, we obtain $Q_{i} \subseteq V_{i}$ satisfying conditions (i) and (ii). 
To establish (iii), notice that the angle between any two lines $p_{i} p_{j}$ and $p_{i}^{\prime} p_{j}^{\prime}\left(p_{i}, p_{i}^{\prime} \in Q_{i}\right.$; $\left.p_{j}, p_{j}^{\prime} \in Q_{j} ; i \neq j\right)$ is $o(1)$. Using the fact that $\left\{V_{1}, V_{j}\right\}$ is $\delta$-regular for all $2 \leq j \leq k+2$, one can recursively pick $p_{j} \in Q_{j}$ so that

$$
\begin{aligned}
\mid\{q \in & \left.Q_{1}: q p_{j} \in E\left(G_{r(i, j)}\right) \text { for all } 2 \leq j \leq k+2\right\} \mid \\
& \geq\left(\frac{\epsilon}{100 k}\right)^{k+1}\left|Q_{1}\right| \geq\left(\frac{\epsilon}{100 k}\right)^{k+6}\left|V_{1}\right| \\
& \geq \frac{1}{F(\delta, k, f)}\left(\frac{\epsilon}{100 k}\right)^{k+6} n .
\end{aligned}
$$

Thus, two elements of this set, $q_{1}$ and $q_{1}^{\prime}$ (say), are relatively far away from each other:

$$
\sqrt{\frac{1}{F(\delta, k, f)}\left(\frac{\epsilon}{100 k}\right)^{k+6} n / 10} \leq d\left(q_{1}, q_{1}^{\prime}\right) \leq \operatorname{diam} Q_{1}=o(1) \min _{j \neq 1} t_{r(1, j)} .
$$

This in turn implies, in the same way as in the proof of Lemma 2.3 (iii), that

$$
\left|\cos \left(\angle q_{1}^{\prime} q_{1} p_{j}\right)\right|=o(1) \quad(2 \leq j \leq k+2)
$$

i.e., every line $p_{1} p_{j}\left(p_{1} \in Q_{1}, p_{j} \in Q_{j}, j \neq 1\right)$ is almost perpendicular to the line $q_{1} q_{1}^{\prime}$. Applying the same argument for $Q_{2}, Q_{3}, \ldots$ (instead of $Q_{1}$ ), we obtain (iii).

Using Lemma 3.1, (i) and $\left|Q_{i}\right|>(\epsilon / 100 k)^{5}\left|V_{1}\right| \geq \delta\left|V_{i}\right|$ we can see (using induction), that there are const $(k, \epsilon, \delta) n^{k+2}(k+2)$-tuples $\left(q_{1}, \ldots, q_{k+2}\right)$, with $q_{i} \in Q_{i}$, such that

$$
d\left(q_{i}, q_{j}\right) \in\left[t_{r(i, j)}, t_{r(i, j)}+c(n)\right]
$$

(For details $c f$. [PA].) Fix one of them. Then repeating the considerations of Lemma 2.4 for this $(k+2)$-tuple only, we get that the assertion of Lemma 2.4 is valid also now. Then the proof of Theorem 2 can be completed in exactly the same way as in the previous section with any function $c(n)=o(\sqrt{n})$. As a matter of fact, in order to apply our argument, it is sufficient to assume that $c(n) \leq c_{k, \epsilon} \sqrt{n}$ for a suitable constant $c_{k, \epsilon}>0$.

\section{References}

[E1] P. Erdős: On sets of distances of $n$ points, Amer. Math. Monthly 53 (1946), 248-250. 
[E2] P. Erdős: On extremal problems for graphs and generalized graphs, Israel J. Math. 2 (1965), 183-190.

[EMPS] P. Erdős, E. Makai, J. Pach and J. Spencer: Gaps in difference sets and the graph of nearly equal distances, in: Applied Geometry and Discrete Mathematics, The Victor Klee Festschrift (P. Gritzmann, B. Sturmfels, eds.), DIMACS Series, Vol. 4, AMS-ACM, 1991, 265-273.

[EP] P. Erdős and G. Purdy: Some extremal problems in combinatorial geometry, in: Handbook of Combinatorics, Springer-Verlag, to appear.

[L] L. Lovász: Combinatorial problems and exercises, Akad. Kiadó, Budapest, North Holland, Amsterdam-New York-Oxford, 1979

[MP] W. Moser and J. Pach: Recent developments in combinatorial geometry, in: New Trends in Discrete and Computational Geometry (J. Pach, ed.), SpringerVerlag, Berlin, 1993, $281-302$.

[PA] J. Pach and P. K. Agarwal: Combinatoral Geometry, J. Wiley, New York, to appear.

[Sz] E. Szemerédi: Regular partitions of graphs, In: Problèmes Combinatoires et Théorie de Graphes, Proc. Colloq. Internat. CNRS, Paris, 1978, 399-401.

[T] P. Turán, Eine Extremalaufgabe aus der Graphentheorie, Mat. Fiz. Lapok 48 (1941), 436-452 (Hungarian, German summary.) 